\title{
利用微波辅助[3+3]环化反应合成稠合吡啶衍生物
}

\author{
吴亚男 $*, a, b$ 杜建宇 $b \quad$ 郝文娟 $b \quad$ 姜 波*,b \\ ( ${ }^{a}$ 南通大学化学化工学院 江苏南通 226019) \\ ( $b$ 江苏师范大学化学与材料科学学院 江苏徐州 221116)
}

\begin{abstract}
摘要 报道了一类新型的微波辅助对甲基苯磺酸促进的 [3+3]环化反应. 利用烯胺酮或烯胺内酯可作为 1,3-双亲核试 剂及炔丙醇可作为 1,3 -双亲电试剂的特性，使其在微波辐射及对甲苯磺酸促进条件下于冰醋酸中在 $70{ }^{\circ} \mathrm{C}$ 反应，实现了 $[3+3]$ 环化反应, 分别区域选择性地合成了 2,2-二芳基取代四氢喹啉-5(1H)-酮衍生物和 2,2-二芳基取代二氢呋喃并 [3,4-b]吡啶-5-酮衍生物，产率良好. 该反应利用微波合成技术促使反应，在短时间内完成(30 min), 唯一副产物为水. 此外，该方法具有原料简单易得、操作简单及底物普适性广等优点，从而为具有潜在应用价值的稠合吡啶骨架的构建 提供了一种绿色、经济且高效的合成策略, 符合绿色化学理念.
\end{abstract}

关键词 微波合成; $[3+3]$ 环化; 喹啉-5(1H)-酮衍生物; 呋喃并 $[3,4-b]$ 吡啶-5-酮衍生物; 绿色化学

\section{Synthesis of Fused Pyridines via Microwave-Assisted $[3+3]$ Cyclization}

\author{
Wu, Yanan*,a,b Du, Jianyu ${ }^{b} \quad$ Hao, Wenjuan $^{b} \quad$ Jiang, Bo ${ }^{*, b}$ \\ ( ${ }^{a}$ School of Chemistry and Chemical Engineering, Nantong University, Nantong, Jiangsu 226019) \\ ( ${ }^{b}$ School of Chemistry \& Materials Science, Jiangsu Normal University, Xuzhou, Jiangsu 221116)
}

\begin{abstract}
A new microwave-assisted $p$-TsOH-promoted [3+3] cyclization was developed. By using the characteristics of enaminones or enamino lactones as 1,3-dinucleaphilic reagents and propargyl alcohols as 1,3-electrophilic reagents, $p$-TsOHpromoted $[3+3]$ cyclization of these substrates at $70{ }^{\circ} \mathrm{C}$ was carried out in acetic acid under microwave irradiation, regioselectively affording 2,2-diaryl-substituted tetrahydroquinoline-5(1H)-ones and 2,2-diaryl-substituted dihydrofuro[3,4- $b]$ pyridin5 -ones in good yields. The reaction can be completed within a short period (30 $\mathrm{min}$ ) by microwave synthetic technology, in which water was the sole by-product. This method features simple and available starting materials, simple operation and wide substrate scope, and provides a green, economic, and efficient synthetic strategy for the construction of fused pyridine skeleton with potential application, which is consistent with the concept of green chemistry.

Keywords microwave synthesis; $[3+3]$ cyclization; quinoline-5 $(1 H)$-one; furo[3,4-b]pyridin-5-one; green chemistry
\end{abstract}

随着人们对环保意识的不断增强, 绿色的合成方法 越来越受到化学工作者的青睐. 微波技术作为绿色合成 方法学的重要组成部分, 为有机合成提供了一种绿色、 经济且高效的合成策略. 微波辅助的有机合成已成为有 机化学领域中的研究热点之一, 这得益于微波辐射下的 有机反应具有反应时间短、产率高和对环境友好等优 点 ${ }^{[1]}$. 微波加热是一种利用磁场加热极性分子的加热方 式, 突破了传统的热传递模式, 其特征表现在两方面: (i)极性分子在微波作用下要按照电磁波的方向进行有
序排列，但分子调整方向速度慢于微波磁场的变换，导 致分子间发生强烈的摩擦, 快速达到很高的温度; (ii)在 微波作用下分子极性发生改变, 迫使电子云分布发生改 变, 对于有些反应有降低能垒的作用 ${ }^{[2]}$. 因此, 与传统 加热方式相比, 微波加热具有极高的效率, 其促进的有 机合成引起了化学工作者的强烈关注 ${ }^{[3]}$. 虽然此研究领 域已取得了进展，继续设计和开发新的微波合成化学仍 然是一类非常值得研究的课题.

含氮杂环化合物不仅存在于天然产物和临床药物

\footnotetext{
* Corresponding author. E-mail: 1498280030@qq.com; jiangchem@jsnu.edu.cn

Received February 4, 2021; revised February 10, 2021; published online February 20, 2021.

Project supported by the National Natural Science Foundation of China (No. 21971090) and the Top-notch Academic Programs Project of Jiangsu Higher Education Institutions.

国家自然科学基金(No. 21971090)和江苏省品牌专业基金资助项目.
} 
结构中 ${ }^{[4]}$, 还被广泛用于农药 ${ }^{[5]}$ 、光电材料 ${ }^{[6]}$ 和有机催化 剂[7]等不同的研究领域. 在含氮杂环化合物中, 喹啉衍 生物是一类非常重要的双环杂环体系, 具有重要的生物 活性, 如抗疮疾 $\left(\mathrm{RCQ}^{[8 \mathrm{a}]}\right.$, Decoquinate $\left.{ }^{[8 \mathrm{~b}]}\right)$ 、抗利什曼虫活 性 (Leishmaniasis) ${ }^{[9]}$ 和抗菌活性 (Nalidixic acid ${ }^{[10 a]}$ 和 Levofloxacin $^{[10 b]}$ 等. 因此, 开发喹啉衍生物的高效合成 具有重要的研究价值. 目前化学工作者已建立了许多用 于合成喹啉衍生物的方法, 包括苯并吡喃-2-酮与芳胺 的反应 ${ }^{[11]}$ 、环-1,3-二酮、丁炔酸酯及芳胺的三组分吡啶

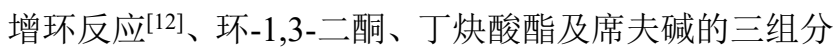
反应 ${ }^{[13]}$ 、环-1,3-二酮、芳胺、醛及氰乙酸乙酯的四组分 反应 ${ }^{[14]}$, 以及环-1,3-二酮、芳胺、丁炔酸酯和靛红或葱 醌的四组分反应 ${ }^{[15]}$ 等. 尽管上述文献报道了喹啉衍生 物的众多合成方法, 然而继续开发新的、绿色环保、廉 价高效的合成策略用于喹啉衍生物的合成仍任重而道 远. 鉴于微波合成化学具有的众多优势 ${ }^{[1-3]}$, 结合我们在 杂环合成方面积累的丰富经验 ${ }^{[16]}$, 本工作利用廉价易 得的环-1,3-二酮衍生的烯胺酮 2 和炔丙醇衍生物 1 作为 原料，在微波辐射下和对甲苯磺酸 $(p-\mathrm{TsOH})$ 促进下，一 步合成 2,2-二芳基取代四氢喹啉-5(1H)-酮衍生物 3, 收 率良好(Scheme 1). 值得一提的是, 用季酮酸衍生的烯 胺内酯 $\mathbf{4}$ 与炔丙醇衍生物 $\mathbf{1}$ 拓展此反应, 该反应进行也 很顺利, 高区域选择性地合成了氢化呋喃并 [3,4- $b]$ 吡 啶-5-酮衍生物 5, 产率优良(Scheme 1). 该反应具有原 料简单易得、反应条件温和、底物普适性广、反应时间 短以及副产物少等优点, 为此类稠合吡啶衍生物的制备 提供了一种绿色、可持续的合成途径.<smiles>CCOC(=O)c1c(C)[nH]c2cc(OCC)c(OCCCOC(=O)C3C(=O)c4ccc(Cc5ccc(OC(F)(F)F)cc5)cc4NC3C)cc2c1=O</smiles>
RCQ Decoquinate<smiles>CCC(CC)C(=O)Nc1cc(OC)cc2c(C)ccnc12</smiles>

图 1 含有喹啉骨架的生物活性分子

Figure 1 Bioactive molecules containing pyridine skeletons

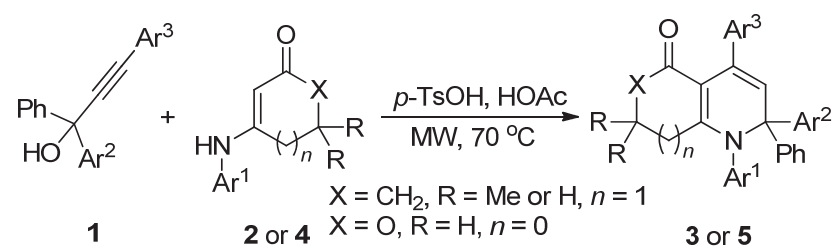

图示 1 稠合吡啶衍生物的微波合成

Scheme 1 Microwave-assisted synthesis of fused pyridines

\section{1 结果与讨论}

\section{1 反应条件的优化}

以炔丙醇衍生物 $1 \mathbf{a}$ 和达咪酮衍生的 $N$-芳基烯胺 $\mathbf{2 a}$ 为模板底物, 研究不同溶剂、促进剂及其用量和不同温 度对产物产率的影响, 实验结果见表 1 . 首先对反应溶 剂进行了笁选(Entries $1 \sim 7)$. 该反应以 1.0 equiv. 的对甲 苯磺酸作为促进剂在微波辐射下和不同溶剂中进行反 应以建立最佳的反应条件. 篮选的反应溶剂包括丙酮、 二氯乙烷(DCE)、二氯甲烷(DCM)、乙腈、甲苯(Toluene)、 乙醇以及冰醋酸。实验结果表明，该反应在冰醋酸中 $100{ }^{\circ} \mathrm{C}$ 时提供目标产物的产率比较高，为 $58 \%$ (Entry 7). 减小对甲苯磺酸的用量导致目标产物的收率降低 (Entry 8), 而增加对甲苯磺酸的用量到 2 equiv. 时也不利 于目标产物的形成(Entry 9). 接下来考察各种促进剂对 反应的影响. 使用三氟化硼乙醚溶液为促进剂, 仅提供 收率为 $37 \%$ 的目标产物(Entry 10). 用其它的布朗斯特 酸如三氟乙酸(TFA)、三氟甲基磺酸(TfOH)给出了类似 的结果(Entries 11 12). 随后对反应温度进行优化发现, 适当降低反应温度有利于该反应的转化(Entries 13 $15)$ ，如在 $70{ }^{\circ} \mathrm{C}$ 时反应能更加有效地合成目标产物，

表 1 化合物 3a 反应条件的优化 ${ }^{a}$

Table 1 Optimization of reaction conditions for the synthesis of 3a
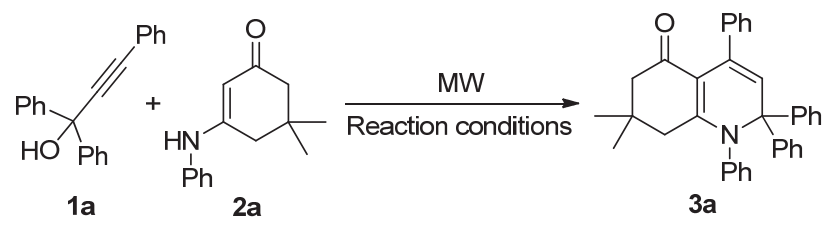

\begin{tabular}{|c|c|c|c|c|}
\hline Entry & $\begin{array}{l}\text { Promotor } \\
\text { (equiv.) }\end{array}$ & Solvent & Temp. $/{ }^{\circ} \mathrm{C}$ & Yield $b / \%$ \\
\hline 1 & $p$-TsOH (1.0) & Acetone & 100 & N.R. \\
\hline 2 & $p$-TsOH (1.0) & DCE & 100 & N.R. \\
\hline 3 & $p$-TsOH (1.0) & DCM & 100 & Trace \\
\hline 4 & $p$-TsOH (1.0) & $\mathrm{MeCN}$ & 100 & 30 \\
\hline 5 & $p$-TsOH (1.0) & Toluene & 100 & Trace \\
\hline 6 & $p$-TsOH (1.0) & $\mathrm{EtOH}$ & 100 & Trace \\
\hline 7 & $p$-TsOH (1.0) & HOAc & 100 & 58 \\
\hline 8 & $p$-TsOH (0.5) & HOAc & 100 & 45 \\
\hline 9 & $p$-TsOH (2.0) & HOAc & 100 & 55 \\
\hline 10 & $\mathrm{BF}_{3} \cdot \mathrm{Et}_{2} \mathrm{O}(1.0)$ & HOAc & 100 & 37 \\
\hline 11 & TFA (1.0) & HOAc & 100 & 21 \\
\hline 12 & TfOH (1.0) & HOAc & 100 & Trace \\
\hline 13 & $p$-TsOH (1.0) & HOAc & 90 & 61 \\
\hline 14 & $p$-TsOH (1.0) & HOAc & 80 & 65 \\
\hline 15 & $p$-TsOH (1.0) & HOAc & 70 & 70 \\
\hline 16 & $p$-TsOH (1.0) & HOAc & 60 & 60 \\
\hline
\end{tabular}

${ }^{a}$ Reaction conditions: propargyl alcohol 1a $(0.2 \mathrm{mmol}), N$-phenyl enaminone 2a $(0.2 \mathrm{mmol})$, promotor (1.0 equiv.), solvent $(3.0 \mathrm{~mL})$, air. ${ }^{b}$ Isolated yield. 
其产率上升到 70\% (Entry 15). 进一步降低反应温度会 使反应不完全, 导致目标产物的收率下降(Entry 16). 因 此最优化反应条件是以对甲苯磺酸为促进剂、冰醋酸为 溶剂和 $70{ }^{\circ} \mathrm{C}$ 为反应温度进行下一步研究.

在最优化条件下, 对该方法的普适性进行了调研 (Scheme 2). 最初以炔丙醇 1a 为代表性底物考察了烯胺 酮化合物 2 上取代基 $\mathrm{Ar}^{1}$ 的适用范围. 结果表明, 多种 与苯环 $\mathrm{Ar}^{1}$ 相连的取代基, 不论其所在芳环位置如对位 或间位以及电子性质如供电子基团 [甲基(1b)、甲氧基 (1c, 1d)] 和吸电子基团[氟(1e)、氯(1f)和(1g)、溴(1h)], 这 些取代基均能有效参与此反应, 从实验结果可以看出, 芳基上基团的电子效应对产物收率有轻微影响, 其中取 代位置相同的供电子基团比吸电子基团表现出更高的 反应效率, 其相应目标产物的收率较高. 芳环 $\mathrm{Ar}^{2}$ 和 $\mathrm{Ar}^{3}$ 分别带有甲基和氯基团的炔丙醇可与不同取代基的 $N$ 芳基烯胺酮在优化条件下顺利进行反应, 转化为相应的 四氢喹啉-5(1H)-酮衍生物 $\mathbf{3 i}$ 和 $\mathbf{3 j}$, 收率分别为 $68 \%$ 和 $50 \%$. 为了继续拓展上述反应, 用 1,3-环已二酮衍生的 烯胺酮 $1 \mathrm{k}$ 和 $1 \mathrm{l}$ 探索该反应的适用范围(Scheme 2). 结果 表明，该类底物在此反应中被证实是一类良好的 1,3-双 亲核试剂, 在微波促进下转化为相应的目标产物 $\mathbf{3 k}$ 和 31, 收率分别为 $61 \%$ 和 $60 \%$.
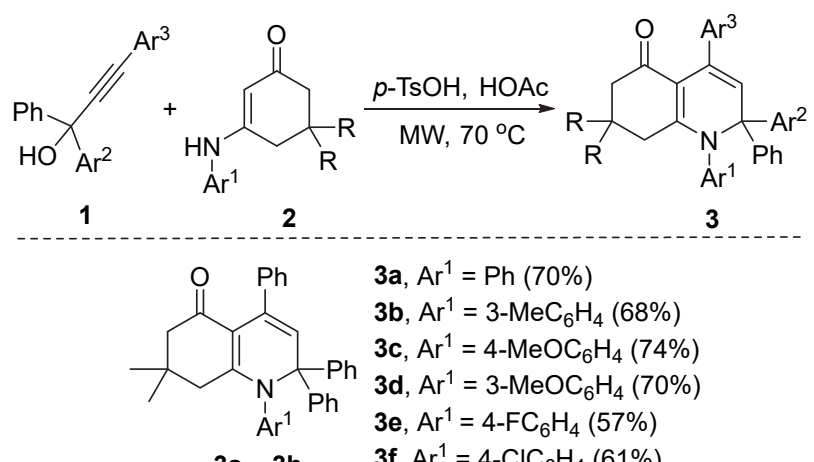

3a, $\mathrm{Ar}^{1}=\mathrm{Ph}(70 \%)$

3b, $\mathrm{Ar}^{1}=3-\mathrm{MeC}_{6} \mathrm{H}_{4}(68 \%)$

3c, $\mathrm{Ar}^{1}=4-\mathrm{MeOC}_{6} \mathrm{H}_{4}(74 \%)$

3d, $\mathrm{Ar}^{1}=3-\mathrm{MeOC}_{6} \mathrm{H}_{4}(70 \%)$

3e, $\mathrm{Ar}^{1}=4-\mathrm{FC}_{6} \mathrm{H}_{4}(57 \%)$

$3 a \sim 3 h$

3f, $\mathrm{Ar}^{1}=4-\mathrm{ClC}_{6} \mathrm{H}_{4}(61 \%)$

3g, $\mathrm{Ar}^{1}=3-\mathrm{ClC}_{6} \mathrm{H}_{4}(60 \%)$

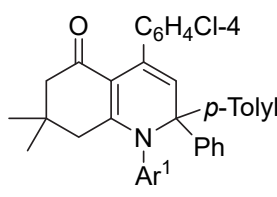

$3 \mathbf{i} \sim 3 \mathbf{j}$

$3 \mathbf{i}, \mathrm{Ar}^{1}=4-\mathrm{MeC}_{6} \mathrm{H}_{4}(68 \%)$

$3 \mathbf{j}, \mathrm{Ar}^{1}=4-\mathrm{BrC}_{6} \mathrm{H}_{4}(50 \%)$

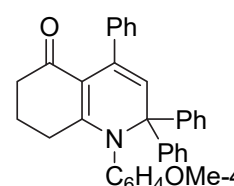

$3 \mathbf{k}(61 \%)$

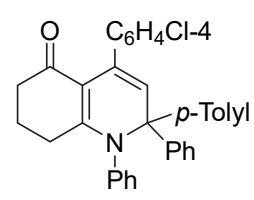

$3 \mathbf{l}(60 \%)$
图示 2 四氢喹啉-5(1H)-酮衍生物 3 的合成

Scheme 2 Synthesis of tetrahydroquinoline-5(1H)-ones 3

完成上述四氢喹啉-5(1H)-酮衍生物的合成后, 我们 考虑用季酮酸衍生的烯胺内酯继续进行上述反应应用 范围的研究(Scheme 3). 值得高兴的是, 反应也能进行 地很顺利, 生成一系列多取代的 2,2-二芳基取代二氢呋 喃并[3,4-b]吡啶-5-酮衍生物 $\mathbf{5 a} \sim \mathbf{5 m}$, 产率为 $62 \%$ $78 \%$. $N$-芳基烯胺内酯的苯环上含有给电子基或吸电子 的取代基均能兼容该反应，其电子效应对反应效率的影 响不明显. 各种官能团如甲基、甲氧基、氟、氯及澳等 都能参与该反应. 调整炔丙醇芳环 $\left(\mathrm{Ar}^{2}\right.$ 与 $\left.\mathrm{Ar}^{3}\right)$ 上的取代 基为甲基或氯时发现，上述取代基均可进行此微波反 应，得到目标产物 $5 \mathrm{~h} \sim 5 \mathrm{~m}$, 产率在 $62 \% \sim 75 \%$ 之间. 所 合成的产物 3 和 5 的结构均经 NMR 谱和 HRMS 表征. 此外，通过单晶 $X$ 射线衍射进一步确定了化合物 $\mathbf{5 a}$ (CCDC: 2060897)的结构(图 1).
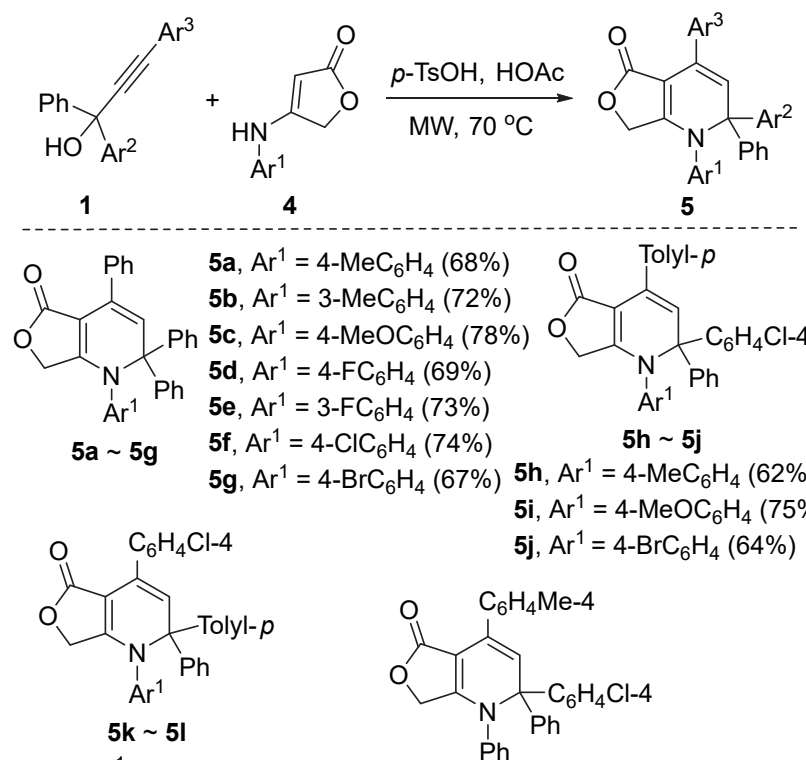

$\mathbf{5 k}, \mathrm{Ar}^{1}=\mathrm{C}_{6} \mathrm{H}_{5}(70 \%)$ 5I, $\mathrm{Ar}^{1}=4-\mathrm{MeC}_{6} \mathrm{H}_{4}(62 \%)$

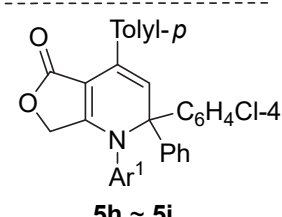

$5 \mathrm{~h}, \mathrm{Ar}^{1}=4-\mathrm{MeC}_{6} \mathrm{H}_{4}(62 \%)$

5i, $\mathrm{Ar}^{1}=4-\mathrm{MeOC}_{6} \mathrm{H}_{4}(75 \%)$

5j, $\mathrm{Ar}^{1}=4-\mathrm{BrC}_{6} \mathrm{H}_{4}(64 \%)$

图示 3 二氢呋喃并 [3,4- $b$ ] 吡啶-5-酮衍生物 5 的合成 Scheme 3 Synthesis of dihydrofuro[3,4- $b]$ pyridin-5-ones 5

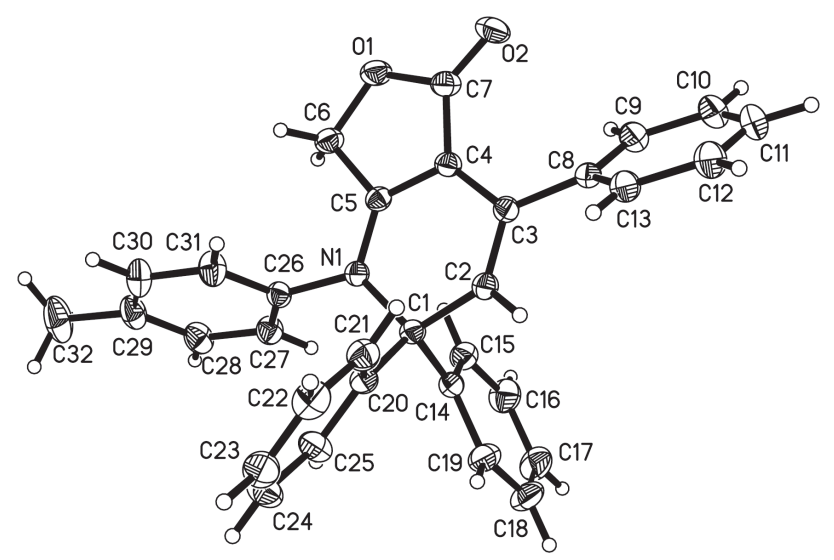

图 2 化合物 $5 \mathbf{a}$ 的晶体结构

Figure 2 Crystal structure of compound 5a

根据文献调研 ${ }^{[17]}$ 以及实验结果, 提出了可能的反 应机理. 如 Scheme 4 所示, 炔丙醇 1 首先在 $p$ - $\mathrm{TsOH}$ 作 用下, 脱水形成碳正离子中间体 $\mathbf{B}$, 经重排成为联烯正 


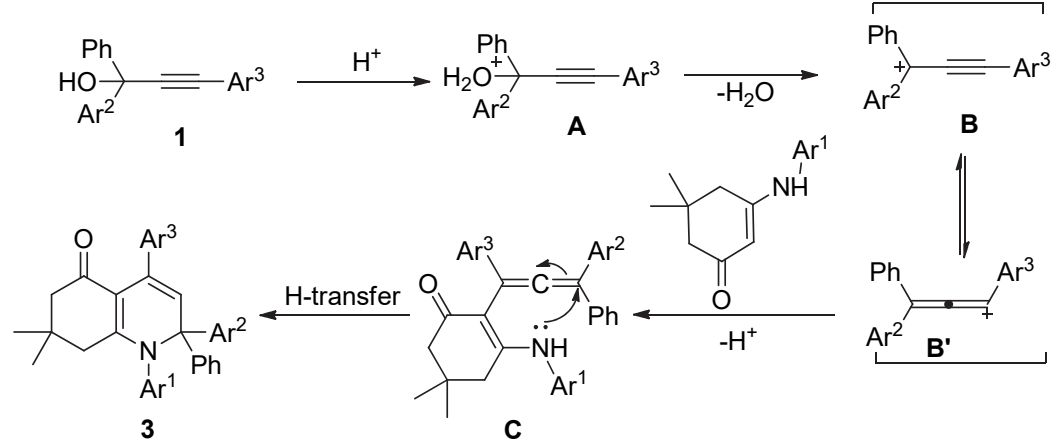

图示 4 可能的反应机理

Scheme 4 Possible reaction mechanism

离子中间体 $\mathbf{B}^{\prime}$. 接下来, 联烯正离子中间体与烯胺酮发 生亲核加成以及随后的分子内环化反应得到最终产物 3. 二氢呋喃并 $[3,4-b]$ 吡啶-5-酮衍生物 5 的形成与上述 过程类似，均实现了炔烃官能化反应 ${ }^{[18]}$. 由于 $p$ - TsOH 是强酸, 易与生成的四氢喹啉-5(1H)-酮衍生物或二氢呋 喃并 $[3,4-b]$ 吡啶-5-酮衍生物成盐而消耗, 因此需要等物 质的量的 $p-\mathrm{TsOH}$.

\section{2 结论}

在微波辐射下, 利用环 1,3-二酮衍生的烯胺酮与炔 丙醇在布朗斯特酸促进下进行脱水 $[3+3]$ 环化反应, 合 成了一系列 2,2-二芳基取代四氢喹啉-5(1H)-酮衍生物, 产率良好. 随后用烯胺内酯作为 1,3-双亲核试剂拓展上 述转化, 同样以较高收率得到一系列新型 2,2-二芳基取 代二氢呋喃并 $[3,4-b]$ 吡啶-5-酮衍生物. 该反应具有原料 廉价易得、反应条件温和、反应时间短以及底物适用范 围宽等优点, 实现了两类吡啶杂环骨架的有效构筑. 使 用微波加速反应过程, 副产物仅为水, 体现了此反应过 程的绿色化.

\section{3 实验部分}

\section{1 仪器与试剂}

XT-5 型熔点测定仪; FTIR-Tensor 27 型红外光谱仪; Bruker microTOF-QII 型质谱仪; Bruker DPX $400 \mathrm{MHz}$ 型核磁共振仪; ZF-20D 型暗箱紫外分析仪; Siemens P4 型四圆 X-单晶衍射仪; 瑞典 Biotage Initiator 型单模微波 合成仪等.

使用的试剂: $\mathrm{CDCl}_{3}-d_{6}$ (内标为 $\mathrm{TMS}$ )、乙酸乙酯(分 析纯)、体积分数为 $95 \%$ 的乙醇(分析纯)、石油醚(分析 纯)、对甲苯磺酸、冰醋酸等.

\section{2 实验方法}

向三个微波反应管中分别加入达米酮 $(1.0 \mathrm{~mol}) 、 1,3-$ 环已二酮 $(1.0 \mathrm{~mol})$ 和季酮酸 $(1.0 \mathrm{~mol})$, 再将芳胺 $(1.0$ $\mathrm{mol}$ )加入三个反应管中, 接下来依次向反应管中倒入 3 $\mathrm{mL}$ 冰醋酸, 搅拌均匀后封盖, 并置于 $120{ }^{\circ} \mathrm{C}$ 微波反应 器中反应 $20 \mathrm{~min}$, 用薄层色谱(TLC)跟踪检测. 待反应 冷却后, 打开反应管. 向体系加入 $5 \mathrm{~mL}$ 水, 经超声振动 产生固体，抽滤后再用体积分数为 $95 \%$ 的乙醇重结晶可 得到纯的烯胺酮 2 和烯胺内酯 4, 表征数据与文献[19] 一致.

向微波反应管中依次加入炔丙醇 $\mathbf{1}(0.2 \mathrm{mmol})$ 、达 米酮衍生的烯胺酮 2(或 1,3-环己二酮衍生的烯胺酮 2) (0.2 mmol)、对甲苯磺酸 $(1.0$ equiv.)和冰醋酸 $(3.0 \mathrm{~mL})$, 然后密封. 将反应管置于微波反应器中 $70{ }^{\circ} \mathrm{C}$ 反应 30 min, 用 TLC 跟踪检测. 待反应完全冷却后, 打开反应 管. 用饱和的 $\mathrm{NaHCO}_{3}$ 溶液和乙酸乙酯(EA)萃取反应体 系. 分离出的有机相先用无水 $\mathrm{Na}_{2} \mathrm{SO}_{4}$ 干燥，再经减压蒸 馏. 采用柱层析 [乙酸乙酯/石油醚 $(V: V=1: 10)$ ]分离 纯化浓缩后的固体混合物，即可得到 2,2-二芳基取代四 氢喹啉- 5(1H)-酮衍生物 3a〜31.

向微波反应管中依次加入炔丙醇 $1(0.2 \mathrm{mmol})$ 、烯 胺内酯 4 ( $0.2 \mathrm{mmol})$ 、对甲苯磺酸 $(1.0$ equiv.)和冰醋酸 $(3$ $\mathrm{mL}$ ), 然后密封. 将反应管置于微波反应器中 $70{ }^{\circ} \mathrm{C}$ 反 应 $30 \mathrm{~min}$, 用 TLC 跟踪检测. 待反应完全冷却后, 打开 反应管. 用饱和的 $\mathrm{NaHCO}_{3}$ 溶液和 $\mathrm{EA}$ 萃取反应体系. 分离出的有机相先用无水 $\mathrm{Na}_{2} \mathrm{SO}_{4}$ 干燥, 再经减压蒸馏. 采用柱层析[乙酸乙酯/石油醚 $(V: V=1: 10)$ ]分离纯化 浓缩后的固体混合物，即可得到 2,2-二芳基取代二氢呋 喃并 [3,4- $b$ ] 吡啶-5-酮衍生物 $\mathbf{5 a} \sim \mathbf{5 m}$.

7,7-二甲基-1,2,2,4-四苯基-2,6,7,8-四氢喹啉-5(1H)酮(3a): 白色固体, 产率 70\%. m.p. 211.9 212.2 ${ }^{\circ} \mathrm{C} ;{ }^{1} \mathrm{H}$ NMR (400 MHz, $\left.\mathrm{CDCl}_{3}\right) \delta: 7.29(\mathrm{~d}, J=6.7 \mathrm{~Hz}, 2 \mathrm{H}, \mathrm{ArH})$, $7.25 \sim 7.12(\mathrm{~m}, 13 \mathrm{H}, \mathrm{ArH}), 7.04(\mathrm{~d}, J=5.0 \mathrm{~Hz}, 3 \mathrm{H}, \mathrm{ArH})$, 6.96 6.92 (m, 2H, ArH), $5.78(\mathrm{~s}, 1 \mathrm{H}, \mathrm{CH}), 2.36(\mathrm{~s}, 2 \mathrm{H}$, $\left.\mathrm{CH}_{2}\right), 2.25$ (s, $\left.2 \mathrm{H}, \mathrm{CH}_{2}\right), 1.08$ (s, $\left.6 \mathrm{H}, \mathrm{CH}_{3}\right) ;{ }^{13} \mathrm{C}$ NMR (100 $\left.\mathrm{MHz}, \mathrm{CDCl}_{3}\right) \delta: 192.1,157.7,144.0,141.7,141.3,135.1$, $129.9,129.0,128.4,127.6,127.5,127.2,126 ., 126.4$, 
124.8, 111.0, 73.1, 51.4, 44.2, 32.9, 28.6; IR (KBr) v: 3125, 2959, 2360, 1639, 1490, 1274, 1058, 767, $702 \mathrm{~cm}^{-1}$; HRMS (ESI) calcd for $\mathrm{C}_{35} \mathrm{H}_{31} \mathrm{NNaO}[\mathrm{M}+\mathrm{Na}]^{+}$504.2303, found 504.2311.

7,7-二甲基-2,2,4-三苯基-1-(间甲苯基)-2,6,7,8-四氢 喹啉-5(1H)-酮(3b): 白色固体, 产率 68\%. m.p. 212.1 $212.4{ }^{\circ} \mathrm{C} ;{ }^{1} \mathrm{H}$ NMR $\left(400 \mathrm{MHz}, \mathrm{CDCl}_{3}\right) \delta: 7.29(\mathrm{~d}, J=6.6$ $\mathrm{Hz}, 2 \mathrm{H}, \mathrm{ArH}), 7.25 \sim 7.14(\mathrm{~m}, 13 \mathrm{H}, \mathrm{ArH}), 6.93$ (t, $J=8.0$ $\mathrm{Hz}, 1 \mathrm{H}, \mathrm{ArH}), 6.84$ (d, $J=7.5 \mathrm{~Hz}, 1 \mathrm{H}, \mathrm{ArH}), 6.75$ (d, $J=$ $7.5 \mathrm{~Hz}, 1 \mathrm{H}, \mathrm{ArH}), 6.65$ (s, 1H, ArH), $5.78(\mathrm{~s}, 1 \mathrm{H}, \mathrm{CH})$, 2.35 (d, J=27.5 Hz, 4H, CH$)_{2}, 2.12\left(\mathrm{~s}, 3 \mathrm{H}, \mathrm{CH}_{3}\right), 1.09$ (s, $\left.6 \mathrm{H}, \mathrm{CH}_{3}\right) ;{ }^{13} \mathrm{C}$ NMR $\left(100 \mathrm{MHz}, \mathrm{CDCl}_{3}\right) \delta: 143.9,138.3$, $130.5,129.1,128.9,128.1,127.7,127.6,127.5,127.2$, $126.8,126.5,124.8,100.0,73.2,51.1,44.3,32.9,28.6$, 21.1; IR (KBr) v: 2956, 2360, 1623, 1488, 1275, 1054, $755,694 \mathrm{~cm}^{-1}$; HRMS (ESI) calcd for $\mathrm{C}_{36} \mathrm{H}_{33} \mathrm{NNaO}[\mathrm{M}+$ $\mathrm{Na}]^{+}$518.2460, found 518.2450.

1-(4-甲氧基苯基)-7,7-二甲基-2,2,4-三苯基-2,6,7,8四氢喹啉-5(1H)-酮 (3c): 白色固体, 产率 74\%. m.p. $221.3 \sim 221.6{ }^{\circ} \mathrm{C} ;{ }^{1} \mathrm{H}$ NMR $\left(400 \mathrm{MHz}, \mathrm{CDCl}_{3}\right) \delta: 7.26 \sim$ 7.21 (m, 6H, ArH), 7.21 7.01 (m, 9H, ArH), 6.83 (d, $J=$ $8.8 \mathrm{~Hz}, 2 \mathrm{H}, \mathrm{ArH}), 6.54$ (d, $J=8.8 \mathrm{~Hz}, 2 \mathrm{H}, \operatorname{ArH}), 5.74$ (s, $1 \mathrm{H}, \mathrm{CH}$ ), 3.67 (s, 3H, $\mathrm{CH}_{3}$ ), 2.33 (s, $\left.2 \mathrm{H}, \mathrm{CH}_{2}\right), 2.23$ (s, $2 \mathrm{H}$, $\left.\mathrm{CH}_{2}\right), 1.08\left(\mathrm{~s}, 6 \mathrm{H}, \mathrm{CH}_{3}\right) ;{ }^{13} \mathrm{C} \mathrm{NMR}\left(100 \mathrm{MHz}, \mathrm{CDCl}_{3}\right) \delta$ : $191.9,158.2,158.1,144.1,141.5,135.0,134.6,130.9$, $129.0,127.6,127.5,127.2,126.4,124.5,113.5,110.4$, 73.2, 55.3, 51.4, 44.2, 32.8, 28.7; IR (KBr) v: 3019, 2944, 1637, 1399, 1251, 1027, 773, $696 \mathrm{~cm}^{-1}$; HRMS (ESI) calcd for $\mathrm{C}_{36} \mathrm{H}_{33} \mathrm{NNaO}_{2}[\mathrm{M}+\mathrm{Na}]^{+} 534.2409$, found 534.2433.

1-(3-甲氧基苯基)-7,7-二甲基-2,2,4-三苯基-2,6,7,8四氢喹啉-5(1H)-酮 (3d): 白色固体, 产率 70\%. m.p. 198.4 198.7 ${ }^{\circ} \mathrm{C} ;{ }^{1} \mathrm{H}$ NMR (400 MHz, $\left.\mathrm{CDCl}_{3}\right) \delta: 7.30$ (s, $2 \mathrm{H}, \mathrm{ArH}), 7.28 \sim 7.02(\mathrm{~m}, 13 \mathrm{H}, \operatorname{ArH}), 6.97(\mathrm{~d}, J=8.0 \mathrm{~Hz}$, $1 \mathrm{H}, \mathrm{ArH}), 6.60(\mathrm{t}, J=8.0 \mathrm{~Hz}, 2 \mathrm{H}, \mathrm{ArH}), 6.45(\mathrm{~s}, 1 \mathrm{H}, \mathrm{ArH})$, 5.79 (s, 1H, CH), 3.63 (s, $\left.3 \mathrm{H}, \mathrm{CH}_{3}\right), 2.43(\mathrm{~d}, J=6.8 \mathrm{~Hz}$, $\left.2 \mathrm{H}, \mathrm{CH}_{2}\right), 2.27$ (s, $2 \mathrm{H}, \mathrm{CH}_{2}$ ), 1.11 (s, $\left.6 \mathrm{H}, \mathrm{CH}_{3}\right) ;{ }^{13} \mathrm{C} \mathrm{NMR}$ $\left(100 \mathrm{MHz}, \mathrm{CDCl}_{3}\right) \delta: 159.4,143.9,142.7,141.3,135.0$, $129.0,127.6,127.5,127.2,126.4,124.9,122.5,115.9$, 112.6, 73.1, 55.3, 51.4, 44.1, 32.9, 28.7, 28.6; IR (KBr) v: 3056, 2963, 1651, 1486, 1270, 1043, 771, $694 \mathrm{~cm}^{-1}$; HRMS (ESI) calcd for $\mathrm{C}_{36} \mathrm{H}_{33} \mathrm{NNaO}_{2}[\mathrm{M}+\mathrm{Na}]^{+}$ 534.2409, found 534.2419.

1-(4-氟苯基)-7,7-二甲基-2,2,4-三苯基-2,6,7,8-四氢 喹啉-5(1H)-酮(3e)：白色固体, 产率 57\%. m.p. 224.4
$224.7{ }^{\circ} \mathrm{C} ;{ }^{1} \mathrm{H}$ NMR $\left(400 \mathrm{MHz}, \mathrm{CDCl}_{3}\right) \delta: 7.29(\mathrm{~s}, 2 \mathrm{H}$, $\operatorname{ArH}), 7.26 \sim 7.13(\mathrm{~m}, 13 \mathrm{H}, \operatorname{ArH}), 6.96 \sim 6.87(\mathrm{~m}, 2 \mathrm{H}$, $\operatorname{ArH}), 6.73(\mathrm{t}, J=8.0 \mathrm{~Hz}, 2 \mathrm{H}, \mathrm{ArH}), 5.77(\mathrm{~s}, 1 \mathrm{H}, \mathrm{CH}), 2.32$ (s, $\left.2 \mathrm{H}, \mathrm{CH}_{2}\right), 2.25\left(\mathrm{~s}, 2 \mathrm{H}, \mathrm{CH}_{2}\right), 1.09\left(\mathrm{~s}, 6 \mathrm{H}, \mathrm{CH}_{3}\right) ;{ }^{13} \mathrm{C}$ NMR (100 MHz, $\left.\mathrm{CDCl}_{3}\right) \delta: 192.1,157.4,143.8,141.2$, $137.7,135.0,131.5,131.4,128.9,128.2,127.9,127.7$, $127.6,127.5,127.3,126.5,124.7,115.4,115.2,111.1$, 73.1, 51.3, 44.2, 32.9, 28.6; IR (KBr) v: 3052, 2930, 2282, 1643, 1390, 1221, 1031, 839, 754, $704 \mathrm{~cm}^{-1}$; HRMS (ESI) calcd for $\mathrm{C}_{35} \mathrm{H}_{30} \mathrm{FNNaO} 522.2209[\mathrm{M}+\mathrm{Na}]^{+}$, found 522.2226 .

1-(4-氯苯基)-7,7-二甲基-2,2,4-三苯基-2,6,7,8-四氢 喹啉-5(1H)-酮(3f): 白色固体, 产率 61\%. m.p. 194.1 $194.3{ }^{\circ} \mathrm{C} ;{ }^{1} \mathrm{H}$ NMR $\left(400 \mathrm{MHz}, \mathrm{CDCl}_{3}\right) \delta: 7.21$ (s, $15 \mathrm{H}$, $\operatorname{ArH}), 6.95$ (d, $J=54.8 \mathrm{~Hz}, 4 \mathrm{H}, \mathrm{ArH}), 5.79(\mathrm{~s}, 1 \mathrm{H}, \mathrm{CH})$, $2.29\left(\mathrm{~d}, J=36.5 \mathrm{~Hz}, 4 \mathrm{H}, \mathrm{CH}_{2}\right), 1.09\left(\mathrm{~s}, 6 \mathrm{H}, \mathrm{CH}_{3}\right) ;{ }^{13} \mathrm{C}$ NMR $\left(100 \mathrm{MHz}, \mathrm{CDCl}_{3}\right) \delta: 143.6,141.1,140.3,135.0$, 132.6, 131.1, 128.9, 128.6, 127.8, 127.5, 127.4, 126.5, 125.1, 73.0, 51.4, 44.3, 33.0, 28.7; IR (KBr) v: 2955, 1637, 1489, 1306, 1091, 741, $700 \mathrm{~cm}^{-1}$; HRMS (ESI) calcd for $\mathrm{C}_{35} \mathrm{H}_{30} \mathrm{ClNNaO}[\mathrm{M}+\mathrm{Na}]^{+}$538.1914, found 538.1910.

1-(3-氯苯基)-7,7-二甲基-2,2,4-三苯基-2,6,7,8-四氢 喹啉-5(1H)-酮 $(3 \mathrm{~g})$ : 白色固体, 产率 $60 \%$. m.p. 202.2 $202.5{ }^{\circ} \mathrm{C}$; ${ }^{1} \mathrm{H}$ NMR $\left(400 \mathrm{MHz}, \mathrm{CDCl}_{3}\right) \delta: 7.30$ (dd, $J=$ 11.9, $4.4 \mathrm{~Hz}, 3 \mathrm{H}, \mathrm{ArH}), 7.26 \sim 7.07$ (m, 12H, ArH), 7.05 $6.98(\mathrm{~m}, 2 \mathrm{H}, \mathrm{ArH}), 6.94$ (s, 1H, ArH), 6.88 (d, $J=7.5 \mathrm{~Hz}$, $1 \mathrm{H}, \mathrm{ArH}), 5.83$ (s, $1 \mathrm{H}, \mathrm{CH}), 2.40$ (s, $\left.2 \mathrm{H}, \mathrm{CH}_{2}\right), 2.28(\mathrm{~s}, 2 \mathrm{H}$, $\left.\mathrm{CH}_{2}\right), 1.12\left(\mathrm{~s}, 6 \mathrm{H}, \mathrm{CH}_{3}\right) ;{ }^{13} \mathrm{C} \mathrm{NMR}\left(100 \mathrm{MHz}, \mathrm{CDCl}_{3}\right) \delta$ : $169.6,163.3,142.1,138.2,135.6,132.4,130.7,129.1$, $128.8,128.3,128.0,127.8,123.5,122.5,94.9,65.1$; IR (KBr) $v: 3052,2959,1641,1488,1303,1031,745,681$ $\mathrm{cm}^{-1}$; HRMS (ESI) calcd for $\mathrm{C}_{35} \mathrm{H}_{30} \mathrm{ClNNaO}[\mathrm{M}+\mathrm{Na}]^{+}$ 538.1914, found 538.1909.

1-(4-溴苯基)-7,7-二甲基-2,2,4-三苯基-2,6,7,8-四氢 喹啉-5(1H)-酮(3h): 白色固体, 产率 64\%. m.p. 189.1 $189.3{ }^{\circ} \mathrm{C} ;{ }^{1} \mathrm{H}$ NMR $\left(400 \mathrm{MHz}, \mathrm{CDCl}_{3}\right) \delta: 7.27(\mathrm{~s}, 1 \mathrm{H}$, ArH), $7.25 \sim 7.15(\mathrm{~m}, 16 \mathrm{H}, \mathrm{ArH}), 6.80(\mathrm{~d}, J=8.4 \mathrm{~Hz}, 2 \mathrm{H}$, $\mathrm{ArH}), 5.78(\mathrm{~s}, 1 \mathrm{H}, \mathrm{CH}), 2.33\left(\mathrm{~s}, 2 \mathrm{H}, \mathrm{CH}_{2}\right), 2.26(\mathrm{~s}, 2 \mathrm{H}$, $\left.\mathrm{CH}_{2}\right), 1.08$ (s, $\left.6 \mathrm{H}, \mathrm{CH}_{3}\right) ;{ }^{13} \mathrm{C}$ NMR $\left(100 \mathrm{MHz}, \mathrm{CDCl}_{3}\right) \delta$ : $143.6,141.1,140.8,135.0,131.5,131.2,128.9,127.8$, $127.5,127.4,126.5,125.1,120.7,120.6,73.0,51.4,44.2$, 33.1, 28.6; IR (KBr) v: 3056, 2956, 1636, 1487, 1281, 1030, 831, 773, $701 \mathrm{~cm}^{-1}$; HRMS (ESI) calcd for $\mathrm{C}_{35} \mathrm{H}_{30^{-}}$ $\mathrm{BrNNaO}[\mathrm{M}+\mathrm{Na}]^{+}$582.1408, found 582.1405.

4-(4-氯苯基)-7,7-二甲基-2-苯基-2-(对甲苯基)- 
2,6,7,8-四氢喹啉-5(1H)-酮(3i): 白色固体，产率 $68 \%$. m.p. $210.3 \sim 210.6{ }^{\circ} \mathrm{C}$; ${ }^{1} \mathrm{H}$ NMR (400 MHz, $\left.\mathrm{CDCl}_{3}\right) \delta$ : $7.25 \sim 7.19(\mathrm{~m}, 4 \mathrm{H}, \operatorname{ArH}), 7.19 \sim 7.13(\mathrm{~m}, 3 \mathrm{H}, \mathrm{ArH})$, $7.13 \sim 7.08(\mathrm{~m}, 4 \mathrm{H}, \mathrm{ArH}), 6.99$ (d, J=8.1 Hz, 2H, ArH), $6.86 \sim 6.77(\mathrm{~m}, 4 \mathrm{H}, \mathrm{ArH}), 5.69(\mathrm{~s}, 1 \mathrm{H}, \mathrm{CH}), 2.33(\mathrm{~s}, 2 \mathrm{H}$, $\left.\mathrm{CH}_{2}\right), 2.28\left(\mathrm{~s}, 3 \mathrm{H}, \mathrm{CH}_{3}\right), 2.23\left(\mathrm{~s}, 2 \mathrm{H}, \mathrm{CH}_{2}\right), 2.20(\mathrm{~s}, 3 \mathrm{H}$, $\left.\mathrm{CH}_{3}\right), 1.07\left(\mathrm{~s}, 6 \mathrm{H}, \mathrm{CH}_{3}\right) ;{ }^{13} \mathrm{C} \mathrm{NMR}\left(100 \mathrm{MHz}, \mathrm{CDCl}_{3}\right) \delta$ : $191.9,158.4,144.0,140.9,140.1,139.0,136.9,133.8$, $132.0,129.5,129.0,128.9,128.8,128.3,127.6,127.2$, 125.0, 110.1, 72.9, 51.4, 44.2, 32.9, 28.7, 28.6, 21.0; IR (KBr) v: 2929, 1614, 1488, 1281, 1062, 777, $704 \mathrm{~cm}^{-1}$; HRMS (ESI) calcd for $\mathrm{C}_{37} \mathrm{H}_{34} \mathrm{ClNNaO}[\mathrm{M}+\mathrm{Na}]^{+}$ 566.2227, found 566.2221.

1-(4-溴苯基)-4-(4-氯苯基)-7,7-二甲基-2-苯基-2-(对 甲苯基)-2,6,7,8-四氢喹啉-5(1H)-酮(3j): 白色固体, 产 率 50\%. m.p. 210.1 210.5 ${ }^{\circ} \mathrm{C} ;{ }^{1} \mathrm{H}$ NMR $(400 \mathrm{MHz}$, $\left.\mathrm{CDCl}_{3}\right) \delta: 7.24 \sim 7.15(\mathrm{~m}, 9 \mathrm{H}, \mathrm{ArH}), 7.11 \sim 7.04(\mathrm{~m}, 4 \mathrm{H}$, ArH), 7.00 (d, $J=7.8 \mathrm{~Hz}, 2 \mathrm{H}, \operatorname{ArH}), 6.80$ (d, $J=7.9 \mathrm{~Hz}$, 2H, ArH), $5.71(\mathrm{~s}, 1 \mathrm{H}, \mathrm{CH}), 2.31\left(\mathrm{~s}, 2 \mathrm{H}, \mathrm{CH}_{2}\right), 2.28(\mathrm{~s}, 3 \mathrm{H}$, $\left.\mathrm{CH}_{3}\right), 2.23\left(\mathrm{~s}, 2 \mathrm{H}, \mathrm{CH}_{2}\right), 1.08\left(\mathrm{~s}, 6 \mathrm{H}, \mathrm{CH}_{3}\right) ;{ }^{13} \mathrm{C}$ NMR (100 $\left.\mathrm{MHz} \mathrm{CDCl}_{3}\right) \delta: 143.6,140.7,137.2,132.2,131.6,131.4$, $128.9,128.8,128.5,127.8,127.6,127.5,125.4,120.7$, 72.8, 33.0, 28.7, 21.0; IR (KBr) v: 2928, 1637, 1487, 1280, $1088,835,806 \mathrm{~cm}^{-1}$; HRMS (ESI) calcd for $\mathrm{C}_{36} \mathrm{H}_{31} \mathrm{Br}-$ $\mathrm{ClNNaO}[\mathrm{M}+\mathrm{Na}]^{+}$563.1175, found 563.1159.

1-(4-甲氧基苯基)-2,2,4-三苯基-2,6,7,8-四氢喹啉5(1H)-酮 (3k): 白色固体，产率 60\%. m.p. 270.1 $270.5{ }^{\circ} \mathrm{C} ;{ }^{1} \mathrm{H}$ NMR (400 MHz, $\left.\mathrm{CDCl}_{3}\right) \delta: 7.27(\mathrm{~s}, 2 \mathrm{H}$, ArH), $7.26 \sim 7.12(\mathrm{~m}, 13 \mathrm{H}, \mathrm{ArH}), 6.82(\mathrm{~d}, J=8.0 \mathrm{~Hz}, 2 \mathrm{H}$, ArH), 6.54 (d, J=8.0 Hz, 2H, ArH), 5.78 (s, 1H, CH), 3.68 $\left(\mathrm{s}, 3 \mathrm{H}, \mathrm{CH}_{3}\right), 2.45 \sim 2.41\left(\mathrm{~m}, 2 \mathrm{H}, \mathrm{CH}_{2}\right), 1.99 \sim 1.94(\mathrm{~m}$, $\left.2 \mathrm{H}, \mathrm{CH}_{2}\right), 1.26\left(\mathrm{~s}, 2 \mathrm{H}, \mathrm{CH}_{2}\right) ;{ }^{13} \mathrm{C} \mathrm{NMR}\left(100 \mathrm{MHz}, \mathrm{CDCl}_{3}\right)$ $\delta$ : 158.3, 143.6, 141.2, 136.7, 134.4, 131.3, 130.8, 130.6, $128.9,127.7,127.5,127.4,127.2,126.5,113.6,101.7$, $100.0,73.3,55.3,37.1,30.6,21.2$; IR (KBr) v: 3123, 1636, 1443, 1248, 1030, 753, $699 \mathrm{~cm}^{-1}$; HRMS (ESI) calcd for $\mathrm{C}_{34} \mathrm{H}_{29} \mathrm{NNaO}_{2}[\mathrm{M}+\mathrm{Na}]^{+}$506.2096, found 506.2088.

4-(4-氯苯基)-1,2-二苯基-2-(对甲苯基)-2,6,7,8-四氢 喹啉-5(1H)-酮(3I): 白色固体, 产率 61\%. m.p. 212.3 $212.5{ }^{\circ} \mathrm{C} ;{ }^{1} \mathrm{H}$ NMR $\left(400 \mathrm{MHz}, \mathrm{CDCl}_{3}\right) \delta: 7.25 \sim 7.20(\mathrm{~m}$, $4 \mathrm{H}, \mathrm{ArH}), 7.18 \sim 7.07(\mathrm{~m}, 7 \mathrm{H}, \mathrm{ArH}), 7.05 \sim 6.91(\mathrm{~m}, 7 \mathrm{H}$, ArH), 5.74 (s, 1H, CH), 2.45 (t, J=8.0 Hz, 2H, $\left.\mathrm{CH}_{2}\right), 2.35$ $\left(\mathrm{t}, J=8.0 \mathrm{~Hz}, 2 \mathrm{H}, \mathrm{CH}_{2}\right), 2.28\left(\mathrm{~s}, 3 \mathrm{H}, \mathrm{CH}_{3}\right), 2.00 \sim 1.93(\mathrm{~m}$, $\left.2 \mathrm{H}, \mathrm{CH}_{2}\right) ;{ }^{13} \mathrm{C} \mathrm{NMR}\left(100 \mathrm{MHz}, \mathrm{CDCl}_{3}\right) \delta: 191.9,160.5$, $143.6,141.5,140.5,139.8,137.0,133.9,132.1,129.9$,
$128.9,128.7,128.5,128.4,127.7,127.6,127.2,125.1$, 110.7, 72.9, 37.2, 30.6, 21.3, 21.0; IR (KBr) v: 3124, 2930, 2360, 1636, 1488, 1022, 778, $701 \mathrm{~cm}^{-1}$; HRMS (ESI) calcd for $\mathrm{C}_{34} \mathrm{H}_{28} \mathrm{ClNNaO}[\mathrm{M}+\mathrm{Na}]^{+}$524.1757, found 524.1739 .

2,2,4-三苯基-1-(对甲苯基)-2,7-二氢呋喃 [3,4- $b$ ]吡 啶-5(1H)-酮(5a)：白色固体，产率 68\%. m.p. 240.9 $241.3{ }^{\circ} \mathrm{C} ;{ }^{1} \mathrm{H}$ NMR $\left(400 \mathrm{MHz}, \mathrm{CDCl}_{3}\right) \delta: 7.48 \sim 7.40(\mathrm{~m}$, $2 \mathrm{H}, \mathrm{ArH}), 7.39 \sim 7.24(\mathrm{~m}, 13 \mathrm{H}, \mathrm{ArH}), 6.89(\mathrm{~d}, J=8.1 \mathrm{~Hz}$, 2H, ArH), 6.72 (d, $J=8.2 \mathrm{~Hz}, 2 \mathrm{H}, \mathrm{ArH}), 5.46$ (s, 1H, CH), 4.49 (s, 2H, $\left.\mathrm{CH}_{2}\right), 2.22$ (s, 3H, $\left.\mathrm{CH}_{3}\right) ;{ }^{13} \mathrm{C} \mathrm{NMR} \mathrm{(100} \mathrm{MHz,}$ $\left.\mathrm{CDCl}_{3}\right) \delta: 169.9,164.3,142.5,138.6,136.5,135.9,129.8$, $129.1,128.9,128.3,128.1,127.9,127.7,123.2,93.6,65.2$, 21.0; IR (KBr) v: 3043, 2936, 2360, 1574, 1471, 1028, $743,697 \mathrm{~cm}^{-1}$; HRMS (ESI) calcd for $\mathrm{C}_{32} \mathrm{H}_{25} \mathrm{NNaO}_{2}[\mathrm{M}+$ $\mathrm{Na}]^{+}$478.1783, found 478.1767 .

2,2,4-三苯基-1-(间甲苯基)-2,7-二氢呋喃 $[3,4-b]$ 吡 啶-5(1H)-酮(5b): 白色固体，产率 72\%. m.p. 265.3 $265.9{ }^{\circ} \mathrm{C} ;{ }^{1} \mathrm{H}$ NMR $\left(400 \mathrm{MHz}, \mathrm{CDCl}_{3}\right) \delta: 7.46 \sim 7.43(\mathrm{~m}$, $2 \mathrm{H}, \mathrm{ArH}), 7.40 \sim 7.26(\mathrm{~m}, 13 \mathrm{H}, \mathrm{ArH}), 7.02 \sim 6.93(\mathrm{~m}, 2 \mathrm{H}$, ArH), 6.74 (d, J=7.6 Hz, 1H, ArH), 6.58 (s, 1H, ArH), $5.49(\mathrm{~s}, 1 \mathrm{H}, \mathrm{CH}), 4.54\left(\mathrm{~s}, 2 \mathrm{H}, \mathrm{CH}_{2}\right), 2.11\left(\mathrm{~s}, 3 \mathrm{H}, \mathrm{CH}_{3}\right) ;{ }^{13} \mathrm{C}$ NMR $\left(100 \mathrm{MHz}, \mathrm{CDCl}_{3}\right) \delta: 169.9,164.1,142.5,139.2$, 139.0 , 135.9, 129.8, 129.3, 129.2, 128.9, 128.3, 128.1, $127.9,127.7,126.0,123.2,93.9,65.2,21.1$; IR (KBr) $v$ : 3056, 2921, 1569, 1441, 1232, 1075, $767 \mathrm{~cm}^{-1}$; HRMS (ESI) calcd for $\mathrm{C}_{32} \mathrm{H}_{25} \mathrm{NNaO}_{2}[\mathrm{M}+\mathrm{Na}]^{+} 478.1783$, found 478.1773 .

1-(4-甲氧基苯基)-2,2,4-三苯基-2,7-二氢呋喃[3,4- $b$ ] 吡啶-5(1H)-酮 $\mathbf{5 c}$ ): 白色固体，产率 78\%. m.p. 239.9 $240.5{ }^{\circ} \mathrm{C} ;{ }^{1} \mathrm{H}$ NMR $\left(400 \mathrm{MHz}, \mathrm{CDCl}_{3}\right) \delta: 7.46 \sim 7.42(\mathrm{~m}$, $2 \mathrm{H}, \mathrm{ArH}), 7.36 \sim 7.26(\mathrm{~m}, 13 \mathrm{H}, \mathrm{ArH}), 6.77 \sim 6.72(\mathrm{~m}, 2 \mathrm{H}$, ArH), $6.61 \sim 6.57(\mathrm{~m}, 2 \mathrm{H}, \mathrm{ArH}), 5.44(\mathrm{~s}, 1 \mathrm{H}, \mathrm{CH}), 4.47$ (s, $\left.2 \mathrm{H}, \mathrm{CH}_{2}\right), 3.70\left(\mathrm{~s}, 3 \mathrm{H}, \mathrm{CH}_{3}\right) ;{ }^{13} \mathrm{C}$ NMR $\left(100 \mathrm{MHz}, \mathrm{CDCl}_{3}\right)$ $\delta: 169.9,164.6,159.2,142.5,135.9,131.7,130.4,129.0$, $128.9,128.3,128.1,127.9,127.7,123.1,114.2$, 93.3, 65.1, 55.4; IR (KBr) v: 3056, 2846, 1470, 1008, 770, $618 \mathrm{~cm}^{-1}$; HRMS (ESI) calcd for $\mathrm{C}_{32} \mathrm{H}_{25} \mathrm{NNaO}_{3}[\mathrm{M}+\mathrm{Na}]$ 494.1732, found 494.1718

1-(4-氟苯基)-2,2,4-三苯基-1,2,5,7-四氢呋喃[3,4- $b]$ 吡啶(5d): 白色固体，产率 69\%. m.p. 253 253.5 ${ }^{\circ} \mathrm{C} ;{ }^{1} \mathrm{H}$ NMR (400 MHz, $\left.\mathrm{CDCl}_{3}\right) \delta: 7.45 \sim 7.42(\mathrm{~m}, 2 \mathrm{H}, \mathrm{ArH})$, $7.37 \sim 7.27$ (m, 13H, ArH), 6.88 6.77 (m, 4H, ArH), 5.48 $(\mathrm{d}, J=1.7 \mathrm{~Hz}, 1 \mathrm{H}, \mathrm{CH}), 4.50\left(\mathrm{~s}, 2 \mathrm{H}, \mathrm{CH}_{2}\right) ;{ }^{13} \mathrm{C} \mathrm{NMR}(100$ $\left.\mathrm{MHz}, \mathrm{CDCl}_{3}\right) \delta: 169.7,163.8,163.2,160.7,142.2,135.7$, 
$135.1,131.1,131.0,129.1,128.8,128.3,128.0,127.9$, 127.8, 123.3, 116.3, 116.1, 94.3, 65.0; IR (KBr) v: 3056, 2349, 1602, 1470, 1280, 1015, 784, $701 \mathrm{~cm}^{-1}$; HRMS (ESI) calcd for $\mathrm{C}_{31} \mathrm{H}_{22} \mathrm{FNNaO}_{2}[\mathrm{M}+\mathrm{Na}]^{+}$482.1532, found 482.1528 .

1-(3-氟苯基)-2,2,4-三苯基-2,7-二氢呋喃 [3,4- $b$ 吡 啶-5(1H)-酮(5e): 白色固体, 产率 73\%. m.p. 232.4 $232.7{ }^{\circ} \mathrm{C} ;{ }^{1} \mathrm{H}$ NMR $\left(400 \mathrm{MHz}, \mathrm{CDCl}_{3}\right) \delta: 7.56 \sim 7.41(\mathrm{~m}$, $2 \mathrm{H}, \mathrm{ArH}), 7.40 \sim 7.22(\mathrm{~m}, 13 \mathrm{H}, \mathrm{ArH}), 7.13 \sim 7.04(\mathrm{~m}, 1 \mathrm{H}$, $\mathrm{ArH}), 6.94 \sim 6.83(\mathrm{~m}, 1 \mathrm{H}, \mathrm{ArH}), 6.74 \sim 6.48(\mathrm{~m}, 2 \mathrm{H}, \mathrm{ArH})$, $5.53(\mathrm{~s}, 1 \mathrm{H}, \mathrm{CH}), 4.58\left(\mathrm{~s}, 2 \mathrm{H}, \mathrm{CH}_{2}\right) ;{ }^{13} \mathrm{C}$ NMR $(100 \mathrm{MHz}$, $\left.\mathrm{CDCl}_{3}\right) \delta: 169.6,163.6,163.2,161.1,142.1,140.7,140.6$, $135.6,130.3,130.2,129.2,128.7,128.3,128.0,127.8$, $124.9,123.6,116.6,116.4,115.8,115.6,95.2,65.1$; IR (KBr) $v: 3055,2359,1628,1465,1015,790,690 \mathrm{~cm}^{-1}$; HRMS (ESI) calcd for $\mathrm{C}_{31} \mathrm{H}_{22} \mathrm{FNNaO}_{2}[\mathrm{M}+\mathrm{Na}]^{+}$ 482.1532, found 482.1518 .

1-(4-氯苯基)-2,2,4-三苯基-2,7-二氢呋喃 [3,4- $b$ 吡 啶-5(1H)-酮 (5f): 白色固体, 产率 74\%. m.p. 248.3 $250.2{ }^{\circ} \mathrm{C} ;{ }^{1} \mathrm{H}$ NMR $\left(400 \mathrm{MHz}, \mathrm{CDCl}_{3}\right) \delta: 7.44 \sim 7.40(\mathrm{~m}$, $2 \mathrm{H}, \operatorname{ArH}), 7.36 \sim 7.27(\mathrm{~m}, 13 \mathrm{H}, \operatorname{ArH}), 7.08(\mathrm{~d}, J=8.5 \mathrm{~Hz}$, $2 \mathrm{H}, \mathrm{ArH}), 6.82$ (d, J=8.5 Hz, 2H, ArH), 5.49 (s, 1H, CH), $4.51\left(\mathrm{~s}, 2 \mathrm{H}, \mathrm{CH}_{2}\right) ;{ }^{13} \mathrm{C} \mathrm{NMR}\left(100 \mathrm{MHz}, \mathrm{CDCl}_{3}\right) \delta: 169.6$, $163.4,142.1,137.7,135.6,134.4,130.4,129.4,129.2$, $128.7,128.3,128.2,128.0,127.8,123.5,94.8,65.0$; IR (KBr) $v: 3035,2933,1625,1491,1209,1059,734,718$ $\mathrm{cm}^{-1}$; HRMS (ESI) calcd for $\mathrm{C}_{31} \mathrm{H}_{22} \mathrm{ClNNaO}_{2}[\mathrm{M}+\mathrm{Na}]^{+}$ 498.1237, found 498.1215.

1-(4-溴苯基)-2,2,4-三苯基-2,7-二氢呋喃 $[3,4-b]$ 吡 啶-5(1H)-酮(5g): 白色固体, 产率 67\%. m.p. 270.2 $270.5{ }^{\circ} \mathrm{C} ;{ }^{1} \mathrm{H}$ NMR $\left(400 \mathrm{MHz}, \mathrm{CDCl}_{3}\right) \delta: 7.45 \sim 7.40(\mathrm{~m}$, $2 \mathrm{H}, \mathrm{ArH}), 7.37 \sim 7.27(\mathrm{~m}, 13 \mathrm{H}, \mathrm{ArH}), 7.23(\mathrm{~d}, J=8.5 \mathrm{~Hz}$, $2 \mathrm{H}, \mathrm{ArH}), 6.75(\mathrm{~d}, J=8.5 \mathrm{~Hz}, 2 \mathrm{H}, \mathrm{ArH}), 5.49(\mathrm{~s}, 1 \mathrm{H}, \mathrm{CH})$, $4.52\left(\mathrm{~s}, 2 \mathrm{H}, \mathrm{CH}_{2}\right) ;{ }^{13} \mathrm{C} \mathrm{NMR}\left(100 \mathrm{MHz}, \mathrm{CDCl}_{3}\right) \delta: 169.6$, $163.3,142.1,138.2,135.6,132.4,130.7,129.1,128.8$, $128.3,128.0,127.8,123.5,122.5,94.9,65.1$; IR (KBr) $v$ : 3095, 2929, 1624, 1467, 1059, $773 \mathrm{~cm}^{-1}$; HRMS (ESI) calcd for $\mathrm{C}_{31} \mathrm{H}_{22} \mathrm{BrNNaO}_{2}[\mathrm{M}+\mathrm{Na}]^{+}$542.0732, found 542.0731 .

2-(4-氯苯基)-2-苯基-1,4-二-对甲苯基-2,7-二氢呋 喃[3,4-b]吡啶-5(1H)-酮(5h): 白色固体, 产率 62\%. m.p. 260.4 260.7 ${ }^{\circ} \mathrm{C} ;{ }^{1} \mathrm{H}$ NMR (400 MHz, $\left.\mathrm{CDCl}_{3}\right) \delta: 7.38 \sim$ $7.26(\mathrm{~m}, 11 \mathrm{H}, \mathrm{ArH}), 7.14$ (d, J=7.9 Hz, 2H, ArH), 6.91 (d, $J=8.2 \mathrm{~Hz}, 2 \mathrm{H}, \mathrm{ArH}), 6.71(\mathrm{~d}, J=8.2 \mathrm{~Hz}, 2 \mathrm{H}, \mathrm{ArH}), 5.37$ (s, $1 \mathrm{H}, \mathrm{CH}), 4.47$ (s, $\left.2 \mathrm{H}, \mathrm{CH}_{2}\right), 2.34$ (s, $\left.3 \mathrm{H}, \mathrm{CH}_{3}\right), 2.24$ (s, $\left.3 \mathrm{H}, \mathrm{CH}_{3}\right) ;{ }^{13} \mathrm{C}$ NMR $\left(100 \mathrm{MHz}, \mathrm{CDCl}_{3}\right) \delta: 169.8,164.2$, $142.3,141.2,138.8,137.8,136.3,133.7,132.8,130.3$, $129.9,129.3,128.8,128.6,128.5,128.2,128.1,127.9$, 122.2, 93.9, 65.1, 21.3, 21.0; IR (KBr) v: 3027, 2923, $1698,1467,1050,846,750 \mathrm{~cm}^{-1}$; HRMS (ESI) calcd for $\mathrm{C}_{33} \mathrm{H}_{26} \mathrm{ClNNaO}_{2}[\mathrm{M}+\mathrm{Na}]^{+}$526.1550, found 526.1547.

2-(4-氯苯基)-1-(4-甲氧基苯基)-2-苯基-4-(对甲苯 基)-2,7-二氢呋喃[3,4-b]吡啶-5(1H)-酮(5i): 白色固体, 产率 75\%. m.p. 263.8 264.3 ${ }^{\circ} \mathrm{C}$; ${ }^{1} \mathrm{H}$ NMR $(400 \mathrm{MHz}$, $\left.\mathrm{CDCl}_{3}\right) \delta: 7.36 \sim 7.26(\mathrm{~m}, 11 \mathrm{H}, \mathrm{ArH}), 7.15(\mathrm{~d}, J=7.9 \mathrm{~Hz}$, $2 \mathrm{H}, \mathrm{ArH}), 6.74(\mathrm{~d}, J=9.0 \mathrm{~Hz}, 2 \mathrm{H}, \mathrm{ArH}), 6.61$ (d, $J=8.9$ $\mathrm{Hz}, 2 \mathrm{H}, \mathrm{ArH}), 5.36$ (s, 1H, CH), 4.46 (s, 2H, $\mathrm{CH}_{2}$ ), 3.72 (s, $\left.3 \mathrm{H}, \mathrm{CH}_{3}\right), 2.34\left(\mathrm{~s}, 3 \mathrm{H}, \mathrm{CH}_{3}\right) ;{ }^{13} \mathrm{C} \mathrm{NMR}\left(100 \mathrm{MHz}, \mathrm{CDCl}_{3}\right)$ $\delta: 169.8,164.5,159.3,142.3,141.2,137.8,133.7,132.8$, $131.5,130.4,129.3,128.8,128.6,128.5,128.3,128.1$, $127.9,125.4,122.4,122.1,114.2,93.5,65.0,55.4,21.5$, 21.3; IR (KBr) v: 3054, 2926, 2358, 1621, 1468, 1246, $1056,814,758 \mathrm{~cm}^{-1}$; HRMS (ESI) calcd for $\mathrm{C}_{33} \mathrm{H}_{26} \mathrm{Cl}-$ $\mathrm{NNaO}_{3}[\mathrm{M}+\mathrm{Na}]^{+}$542.1499, found 542.1485.

1-(4-溴苯基)-2-(4-氯苯基)-2-苯基-4-(对甲苯基)2,7-二氢呋喃 $[3,4-b]$ 吡啶-5 $(1 H)$-酮 $(\mathbf{5 j})$ : 白色固体, 产率 64\%. m.p. $244.8 \sim 245.7{ }^{\circ} \mathrm{C} ;{ }^{1} \mathrm{H}$ NMR (400 MHz, $\mathrm{CDCl}_{3}$ ) $\delta: 7.41 \sim 7.26(\mathrm{~m}, 12 \mathrm{H}, \operatorname{ArH}), 7.25(\mathrm{~s}, 1 \mathrm{H}, \operatorname{ArH}), 7.15(\mathrm{~d}$, $J=7.9 \mathrm{~Hz}, 2 \mathrm{H}, \mathrm{ArH}), 6.74(\mathrm{~d}, J=8.5 \mathrm{~Hz}, 2 \mathrm{H}, \mathrm{ArH}), 5.40$ (s, $1 \mathrm{H}, \mathrm{CH}), 4.50\left(\mathrm{~s}, 2 \mathrm{H}, \mathrm{CH}_{2}\right), 2.34\left(\mathrm{~s}, 3 \mathrm{H}, \mathrm{CH}_{3}\right) ;{ }^{13} \mathrm{C}$ NMR $\left(100 \mathrm{MHz}, \mathrm{CDCl}_{3}\right) \delta: 169.5,163.2,141.8,140.8$, $138.0,134.0,132.6,132.5,130.6,130.1,129.3,128.6$, $128.5,128.4,128.2,128.1,122.7,122.5,95.1,65.0,21.3$; IR (KBr) v: 3056, 2919, 1620, 1487, 1092, 810, $727 \mathrm{~cm}^{-1}$; HRMS (ESI) calcd for $\mathrm{C}_{32} \mathrm{H}_{23} \mathrm{BrClNNaO}_{2}[\mathrm{M}+\mathrm{Na}]^{+}$ 590.0498 , found 590.0488 .

4-(4-氯苯基)-1,2-二苯基-2-(对甲苯基)-2,7-二氢呋 喃[3,4-b]吡啶-5(1H)-酮(5k): 白色固体, 产率 70\%. m.p. $276.5 \sim 277.1{ }^{\circ} \mathrm{C} ;{ }^{1} \mathrm{H}$ NMR $\left(400 \mathrm{MHz}, \mathrm{CDCl}_{3}\right) \delta: 7.37(\mathrm{~d}$, $J=8.5 \mathrm{~Hz}, 2 \mathrm{H}, \mathrm{ArH}), 7.35 \sim 7.26(\mathrm{~m}, 7 \mathrm{H}, \mathrm{ArH}), 7.21(\mathrm{~d}$, $J=8.1 \mathrm{~Hz}, 2 \mathrm{H}, \operatorname{ArH}), 7.16(\mathrm{~d}, J=7.3 \mathrm{~Hz}, 1 \mathrm{H}, \operatorname{ArH})$, $7.13 \sim 7.08(\mathrm{~m}, 4 \mathrm{H}, \mathrm{ArH}), 6.86(\mathrm{~d}, J=7.7 \mathrm{~Hz}, 2 \mathrm{H}, \operatorname{ArH})$, $5.43(\mathrm{~s}, 1 \mathrm{H}, \mathrm{CH}), 4.50\left(\mathrm{~s}, 2 \mathrm{H}, \mathrm{CH}_{2}\right), 2.34\left(\mathrm{~s}, 3 \mathrm{H}, \mathrm{CH}_{3}\right) ;{ }^{13} \mathrm{C}$ NMR $\left(100 \mathrm{MHz}, \mathrm{CDCl}_{3}\right) \delta: 169.8,164.1,142.4,139.4$, $139.1,137.7,134.3,133.8,129.6,129.2,129.1,128.9$, $128.8,128.6,128.5,128.1,128.0,127.9,127.8,123.5$, 93.5, 65.2, 21.1; IR (KBr) v: 3055, 2917, 1623, 1493, $1014,813,788 \mathrm{~cm}^{-1}$; HRMS (ESI) calcd for $\mathrm{C}_{32} \mathrm{H}_{24} \mathrm{Cl}-$ $\mathrm{NNaO}_{2}[\mathrm{M}+\mathrm{Na}]^{+}$512.1393, found 512.1390.

4-(4-氯苯基)-2-苯基-1,2-二-对甲苯基-2,7-二氢呋 
喃[3,4-b]吡啶-5(1H)-酮(5l): 白色固体, 产率 62\%. m.p. 273 273.2 ${ }^{\circ} \mathrm{C} ;{ }^{1} \mathrm{H}$ NMR (400 MHz, $\left.\mathrm{CDCl}_{3}\right) \delta: 7.37$ (d, $J=8.4 \mathrm{~Hz}, 2 \mathrm{H}, \mathrm{ArH}), 7.35 \sim 7.26(\mathrm{~m}, 7 \mathrm{H}, \mathrm{ArH}), 7.21(\mathrm{~d}$, $J=8.1 \mathrm{~Hz}, 2 \mathrm{H}, \mathrm{ArH}), 7.11$ (d, $J=8.1 \mathrm{~Hz}, 2 \mathrm{H}, \mathrm{ArH}), 6.89$ (d, $J=8.2 \mathrm{~Hz}, 2 \mathrm{H}, \mathrm{ArH}), 6.71(\mathrm{~d}, J=8.0 \mathrm{~Hz}, 2 \mathrm{H}, \mathrm{ArH})$, $5.41(\mathrm{~s}, 1 \mathrm{H}, \mathrm{CH}), 4.48\left(\mathrm{~s}, 2 \mathrm{H}, \mathrm{CH}_{2}\right), 2.35\left(\mathrm{~s}, 3 \mathrm{H}, \mathrm{CH}_{3}\right)$, $2.23\left(\mathrm{~s}, 3 \mathrm{H}, \mathrm{CH}_{3}\right) ;{ }^{13} \mathrm{C} \mathrm{NMR}\left(100 \mathrm{MHz}, \mathrm{CDCl}_{3}\right) \delta: 169.9$, $164.5,142.4,139.5,138.6,137.6,136.4,134.4,133.7$, $129.8,129.6,128.9,128.8,128.7,128.1,127.9,127.8$, 123.4, 93.1, 65.2, 21.1, 21.0; IR (KBr) v: 3031, 2918, 1621, 1467, 1213, 1053, 836, $747 \mathrm{~cm}^{-1}$; HRMS (ESI) calcd for $\mathrm{C}_{33} \mathrm{H}_{26} \mathrm{ClNNaO}_{2}[\mathrm{M}+\mathrm{Na}]^{+}$526.1550, found 526.1533 .

2-(4-氯苯基)-1,2-二苯基-4-(对甲苯基)-2,7-二氢呋 喃[3,4- $b]$ 吡啶-5(1H)-酮 $(5 \mathrm{~m})$ : 白色固体, 产率 $68 \%$. m.p. 213.3 $213.7{ }^{\circ} \mathrm{C} ;{ }^{1} \mathrm{H}$ NMR (400 MHz, $\left.\mathrm{CDCl}_{3}\right) \delta: 7.34 \sim$ $7.27(\mathrm{~m}, 10 \mathrm{H}, \mathrm{ArH}), 7.26 \sim 7.09(\mathrm{~m}, 6 \mathrm{H}, \operatorname{ArH}), 6.86(\mathrm{~d}, J=$ $7.8 \mathrm{~Hz}, 2 \mathrm{H}, \mathrm{ArH}), 5.40(\mathrm{~s}, 1 \mathrm{H}, \mathrm{CH}), 4.50\left(\mathrm{~s}, 2 \mathrm{H}, \mathrm{CH}_{2}\right)$, $2.35\left(\mathrm{~s}, 3 \mathrm{H}, \mathrm{CH}_{3}\right) ;{ }^{13} \mathrm{C} \mathrm{NMR}\left(100 \mathrm{MHz}, \mathrm{CDCl}_{3}\right) \delta: 169.7$, $163.8,142.2,141.1,139.0,137.9,133.8,132.7,130.3$, $129.4,129.3,129.1,128.8,128.7,128.6,128.5,128.3$, 128.1, 127.9, 122.3, 94.3, 65.1, 21.3; IR (KBr) v: 3056, 2915, 1625, 1466, 1205, 1053, 827, $756 \mathrm{~cm}^{-1}$; HRMS (ESI) calcd for $\mathrm{C}_{32} \mathrm{H}_{24} \mathrm{ClNNaO}_{2}[\mathrm{M}+\mathrm{Na}]^{+}$512.1393, found 512.1389 .

辅助材料(Supporting Information) 化合物 $\mathbf{3 a} \sim \mathbf{3 1}$ 、 5a $\sim 5 \mathbf{m}$ 的 ${ }^{1} \mathrm{H}$ NMR 和 ${ }^{13} \mathrm{C}$ NMR 图谱. 这些材料可以免 费从本刊网站(http://sioc-journal.cn/)上下载.

\section{References}

[1] (a) Gan, L.; Wei, L.; Wan, J. P. ChemistrySelect 2020, 5, 7822. (b) Xiong, J.; Zhong, G. F.; Liu, Y.-Y. Adv. Synth. Catal. 2019, 361, 550 .

(c) Wang, L.; Shi, L.-X.; Liu, L.; Li, Z.-X.; Xu, T.; Hao, W.-J.; Li, G.; Tu, S.-J.; Jiang, B. J. Org. Chem. 2017, 82, 3605.

(d) Wu, Y.; Lin, Y. W.; He, W. M. Chin. Chem. Lett. 2020, 31, 2999. (e) Li, W.; Shu, L.; Wang, Q.; Li, G. Y.; Shan, Y. Chin. J. Org. Chem. 2019, 39, 1976 (in Chinese).

(李蔚, 宿亮, 汪秋安, 李高阳, 单杨, 有机化学, 2019, 39, 1976.)

[2] (a) Bose, A. K.; Manhas, M. S.; Ghosh, M.; Shah, M.; Raju, V. S.; Bari, S. S.; Newaz, S. N.; Banik, B. K.; Chaudhary, A. G.; Barakat, K. J. J. Org. Chem. 1991, 56, 6968.

(b) Baghurst, D. R.; Mingos, D. M. P. J. Organomet. Chem. 1990, 384, 57.

(c) Raner, K. D.; Strauss, C. R. J. Org. Chem. 1992, 57, 6231.

(d) Caddick, S. Tetrahedron 1995, 51, 10403.

[3] (a) Sheng, J.; Wang, Y.; Su, X. Angew. Chem., Int. Ed. 2017, 56, 4824 .

(b) Lin W.; Zhuang, C. W.; Hu, X. X. Chin. J. Org. Chem. 2020, 40, 408 (in Chinese).

(林伟, 庄苍伟, 胡秀秀, 有机化学, 2020, 40, 408.)

(c) Zeng, R.; Shan, C.; Liu, M. Org. Lett. 2019, 21, 2312. (d) Lan, X.-C.; Chen, T.-T.; Zhao, Y.; Wu, Y.; Wang, J.; Tu, S.-J.; Jiang, B.; Hao, W.-J. Tetrahedron Lett. 2017, 58, 1519.

(e) Wang, A.-F.; Zhou, P.; Zhu, Y.-L.; Hao, W. J.; Li, G.; Tu, S.-J.; Jiang, B. Chem. Commun. 2017, 53, 3369.

(f) Jiang, B.; Zhang, T.-S.; Fu, R.; Hao, W.-J.; Wang, S.-L.; Tu, S.-J. Tetrahedron 2016, 72, 5652.

(g) Fan, W.; Li, Y.-R.; Li, Q.; Jiang, B.; Li, G. Tetrahedron 2016 $72,4867$.

(h) Hao, W. J.; Zhou, P.; Wu, F. Y.; Jiang, B.; Tu, S. J.; Li, G. Eur. J. Org. Chem. 2016, 2016, 1968.

[4] Kumar, D.; Jain, S. K. Curr. Med. Chem. 2016, 23, 4338.

[5] (a) Mahmoodi, N. M.; Arami, M.; Limaee, N. Y.; Gharanjig, K. J. Hazand. Mater. 2007, 145, 65.

(b) Amuti, K.; Trombini, A.; Giammamusti, L.; Sbriscia, C.; Harder, H.; Gabard, J. Brighton Crop Prot. Conf.--Weeds 1997, 1, 59.

(c) Ma, Q.; Zhang, X.; Qu, Y. Front. Microbiol. 2018, 9, 2625.

[6] (a) Chen, D.; Su, S. J.; Cao, Y. J. Mater. Chem. 2014, $2,9565$.

(b) Domcke, W.; Ehrmaier, J.; Sobolewski, A. L. ChemPhotoChem 2019, 3, 10.

[7] (a) Bhunia, A.; Biju, A. T. Synlett 2014, 25, 608.

(b) Kuhl, O. Coord. Chem. Rev. 2004, 248, 411.

[8] (a) Cowley, R.; Leung. S.; Fisher, N.; Al-Helal, M.; Berry, N. G.; Lawrenson, A. S.; Sharma, R.; Shone, A. E.; Ward, S. A.; Biagini, G. A.; O'Neill, P. M. MedChemComm 2012, 3, 39.

(b) Da Cruz, F. P.; Martin, C.; Buchholz, K.; Lafuente-Monasterio, M. J.; Rodrigues, T.; Sonnichsen, B.; Moreira, R.; Gamo, F. J.; Marti, M.; Mota, M. M.; Hannus, M.; Prudencio, M. J. Infect. Dis. 2012, 205, 1278.

[9] Reynolds, K. A.; Loughlin, W. A.; Young, D. J. Mini-Rev. Med. Chem. 2013, 13, 730 .

[10] (a) Bisacchi, G. S. J. Med. Chem. 2015, 58, 4874.

(b) Sissi, C.; Palumbo, M. Curr. Med. Chem.: Anti-Cancer Agents 2003, 3, 439 .

[11] (a) Oehldrich, J.; Cook, J. M. J. Org. Chem. 1977, 42, 889. (b) Yan, W. T. Org. Biomol. Chem. 2018, 16, 9440.

[12] (a) Sarkar, R.; Mukhopadhyay, C. Tetrahedron Lett. 2014, 55, 2618. (b) Sarkar, R.; Mukhopadhyay, C. Tetrahedron Lett. 2018, 59, 3069.

[13] Sun, J.; Sun, Y.; Gao, H.; Yan, C.-G. Eur. J. Org. Chem. 2011, 2011, 6952.

[14] Ahmed, N. S.; Badahdah, K. O.; Qassar, H. M. Med. Chem. Res. 2017, 26, 1201.

[15] (a) Sun, J.; Sun, Y.; Gao, H.; Yan, C.-G. Eur. J. Org. Chem. 2012, $2012,1976$.

(b) Debnath, K.; Pramanik, A. Tetrahedron Lett. 2015, 56, 1654.

(c) Nikoofar, K.; Khani, S. Catal. Lett. 2018, 148, 1651.

(d) Zhu, D.; Sun, J.; Yan, C.-G. J. Heterocycl. Chem. 2016, 53, 583.

[16] (a) Li, M.; Wang, R.; Hao, W.; Jiang, B. Chin. J. Org. Chem. 2020, 40, 1540 (in Chinese).

(李梦帆, 王榕, 郝文娟, 姜波, 有机化学, 2020, 40, 1540.)

(b) Wang, R.; Xu, L.; Lu, Y.; Jiang, B.; Hao, W. Chin. J. Org. Chem. 2021, 41, 1582 (in Chinese)

(王榕, 徐立晨，卢逸，姜波，郝文娟，有机化学，2021，41，1582.) (c) Li, Q.; Li, M.; Shi, S.; Ji, X.; He, C.; Jiang, B.; Hao, W. Chin. J. Org. Chem. 2020, 40, 384 (in Chinese).

(李庆雪，李梦伟，时绍青，季晓霜，何春兰，姜波，郝文娟，有 机化学, 2020, 40, 384.)

(d) Wang, D.; Wang, S.-C.; Hao, W.-J.; Tu, S.-J.; Jiang, B. Chin. J. Chem. 2021, 39, 106

(e) Zuo, H.-D.; Hao, W.-J.; Zhu, C.-F.; Guo, C.; Tu, S.-J.; Jiang, B. Org. Lett. 2020, 22, 4471.

[17] (a) Gan, L.; Yu, Q.; Liu, Y. Y.; Wan, J. P. J. Org. Chem. 2021, 86 1231.

(b) Fu, L. Q.; Xu, Z. R.; Wan, J. P.; Liu, Y. Y. Org. Lett. 2020, 22, 9518.

(c) Yu, Q.; Liu, Y. Y.; Wan, J. P. Org. Chem. Front. 2020, 7, 2770.

(d) Luo, T.; Wan, J. P.; Liu, Y. Y. Org. Chem. Front. 2020, 7, 1107.

(e) Wang, G. D.; Guo, Y. H.; Wan, J. P. Chin. J. Org. Chem. 2020 
40, 645 (in Chinese).

(王国栋，郭艳辉，万结平，有机化学, 2020, 40, 645.)

(f) Liu, Y. Y.; Xiong, J.; Wan, J. P. Adv. Synth. Catal. 2020, 362, 877.

[18] (a) Gui, Q. W.; He, X. L.; Wang, W. J.; Zhou, H. L.; Dong, Y. M.; Wang, N. Q.; Tang, J. X.; Cao, Z.; He, W. M. Green Chem. 2020, 22,118 .

(b) Wang, Z.; He, W. M. Chin. J. Org. Chem. 2019, 39, 3594 (in Chinese).

(王峥，何卫民，有机化学， 2019, 39, 3594.)

(c) Wu, Y. Q.; He, W. M. Chin. J. Org. Chem. 2020, 40, 2597 (in Chinese).
(吴燕，何卫民，有机化学，2020, 40, 2597.)

(d) Zhang, P.; Shi, H. N.; Zhang, T. S.; Cai, P. J.; Jiang, B.; Tu, S. J. Chin. J. Org. Chem. 2020, 40, 423 (in Chinese).

(张萍, 石浩楠, 张天舒, 蔡佩君, 姜波, 屠树江, 有机化学, 2020, 40, 423.)

[19] (a) Bandgar, B. P.; Patil, S. A.; Korbad, B. L.; Bandgar, S. B.; Hote, B. S. Aust. J. Chem. 2008, 61, 552

(b) Matam, S. K. P.; Perumal, M. S. ChemistrySelect 2017, 2, 2363.

(c) da Rocha Pissurno, A. P.; da Silva de Laurentiz, R. Synth. Commun. 2017, 47, 1874.

(d) Takefumi, M.; Naoki, T.; Takafumi, N.; Yumi, T. Heterocycles 1988, 27, 1907.

(Cheng, F.) 